\title{
Variable Kernel Bandwidth Tracking Algorithm Based on Contourlet Histogram and Information Entropy
}

\author{
Rui $\mathrm{Li}^{1}$ and Wen Wang ${ }^{2}$ \\ ${ }^{1,2}$ School of Computer and Communication, LanZhou University of Technology, \\ LanZhou 730050, GanSu, China \\ 'lirui@nwnu.edu.cn, ${ }^{2}$ cicichun@163.com
}

\begin{abstract}
In order to solve the problem that traditional mean shift tracking algorithm based on color histogram is easily affected by similar color non-target object and the change of the size of target, a variable kernel bandwidth tracking algorithm is put forward based on contourlet histogram and information entropy. The target is modeled by contourlet histogram, as iterative parameters in mean shift algorithm, and according to the relationship between information entropy and the size of target, a scale-update item is added to mean shift algorithm to track the target that has a change in size. Experimental results show that the proposed tracking algorithm can not only effectively overcome the interference of similar color non-target object in the scene, but also adapt to the changes in target's size stably, and this algorithm had a good robustness.
\end{abstract}

Keywords: contourlet histogram; information entropy; mean shift; variable kernel bandwidth

\section{Introduction}

Visual target tracking is a hot topic in the field of computer vision, providing an important source for the analysis of interested objects' movement behavior in the scene. Target tracking is widely used in security monitoring, intelligent transportation, military guidance and human-computer interaction, etc. The current tracking algorithms are mostly based on feature of color histogram, which is easy to acquire and to calculate the similarity. To some degree, it can resist deformation of the targets. However, ordinary color histogram only uses the statistical characteristics of image pixels and doesn't provide spatial information of the image, so the tracking result is generally not satisfactory when the target is affected by illumination change or similar color non-target object[1].

Textural features are very important features in image processing and pattern recognition, with some good properties such as resisting the illumination mutations, good local sequentiality [2-3]. They also have rotation invariance, and a strong ability to resist noise. In 2002, a new non-adaptive multiscale transform - contourlet transform [4] - was proposed by Minh N. Do and Martin Vetterli, which is a multiresolution, local and multidirectional image representation method. The image can be decomposed at any direction and at any scale by contourlet transform, and this transform has the advantage of describing the contour and texture information of the image.

On the basis of contourlet transform on image, the concept of contourlet histogram was proposed in literature [5]. The histogram was reconstructed according to coefficients of each sub-band through contourlet transform decomposition, thus improving the rotational invariance so that the histogram could be used for extracting image texture information. Literature [6] used contourlet histogram to extract the target 
images' textural feature, took the histogram as target model, and tracked the target by mean shift tracking algorithm. Contourlet histogram and spatial histogram were combined with mean shift tracking algorithm to track the target in literature [7]. When the target is in partial occlusion, good tracking effect could still be obtained by the two methods. As the size of kernel bandwidth is invariable, the tracking effect is not satisfying when the target has change in size.

When the target is in the process of movement, the size of target is not always constant, which requires that the size of tracking window change with the change of the size of target. Because the kernel bandwidth isn't adjusted in traditional mean shift tracking algorithm, many researchers proposed some algorithms that update the size of tracking window to solve this problem. Comaniciu D [8] et al put forward a method to adjust the size of tracking window by the increments of plus or minus $10 \%$ on the basis of original kernel window, then the best kernel bandwidth was selected according to the biggest Bhattacharyya coefficient in three iterative tracking. The method only works when the size of target decreases, and the increments of plus or minus $10 \%$ can only reflect changes in the target's size qualitatively, can not reflect changes in the target's size accurately. Qian [9] et al introduced a multiscale image information measure method in target tracking, which used the changes of target image information to adjust the size of tracking window, then the method was applied to other tracking algorithms by S.A.Mohammadi [10], Q.Lin [11] et al, and good effect were obtained, though it was very complicated to extract and calculate the image information. The distance between the block candidate target tracking window's center and the centroid of target was adopted to adjust the size of tracking window in literature [12], however, it was complex to decide the change of target's size.

In this paper, contourlet histogram is taken as iterative parameters to track the target with mean shift algorithm, and by means of the relationship between information entropy and the size of target, an item is added that can update the kernel bandwidth in mean shift algorithm. Consequently, a variable kernel bandwidth tracking algorithm is put forward based on contourlet histogram and information entropy, in order to overcome the disadvantages that color histogram is easily affected by similar color non-target object and the tracking effect is not satisfying when the target's size changes.

\section{Contourlet Transform and Textural Feature Extraction}

\subsection{Introduction of Contourlet Transform}

Contourlet transform is also known as Pyramidal Direction Filter Bank (PDFB). Its basic idea is to use a similar wavelet multiscale decomposition to capture edge singularity, then the transform brings singular points that are in near position together into contour segments according to the directional information. To achieve contourlet transform, two separate steps need be completed: Laplace Pyramid (LP) decomposition and Directional Filter Bank (DFB) filter.

Contourlet transform firstly uses LP filter to do the multiscale image decomposition, in order to capture the singular points in images. One LP decomposition does low-pass filtering to original signal, and it divides the original image signal into a downsampling low-frequency component and a high-frequency component that is the difference between original signal and low-frequency sampling signal. And then we continue the decomposition to the low-frequency components, thus the multiresolution of image can be realized. Compared with the critical sampling wavelet, each level of LP decomposition only generates one band-pass image in the high-dimensional case, so that the phenomenon of frequency aliasing are avoided [13-14]. DFB are applied to each level of the high-frequency components which LP decomposed, thus $2^{n}$ directional sub-bands can be got in any scale. Figure 1 shows the structure schematic of 
the discrete contourlet transform filter banks. In this paper, LP uses "9-7", which is a linear phase satisfies the orthogonality, because "9-7" is suit for processing the image; DFB uses "pkva6".

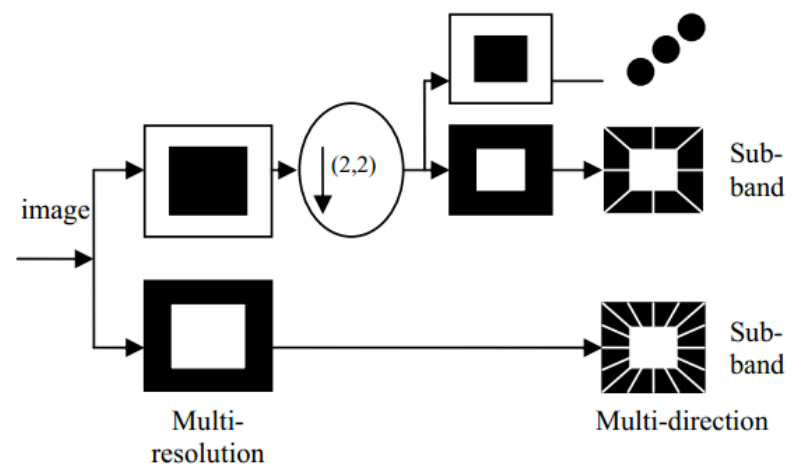

Figure 1. Contourlet Transform Structure Schematic

\subsection{Contourlet Histogram}

A series of sub-images are obtained after the image processed by contourlet transform. At each scale, we set the direction of sub-image that owns maximal energy in all sub-images as the principal direction, and the corresponding component should be arranged in the first place, the other components should be arranged in descending order according to their energy parameter values. When the target image has a change of rotation, but it still has the same principal direction. When the histogram is constructed, the sub-image is also arranged in the same position, thus textural rotation invariance of the target image is enhanced. Contourlet histogram can be constructed as follows [5]:

(1) A 2-level contourlet decomposition and a 3-level contourlet decomposition are processed to original image, then 4 and 8 sub-images $w_{k}$ are obtained respectively at two different scales.

(2) For contourlet coefficient matrix of each sub-image, the energy $E_{k}$ is calculated by formula (1), in order to adjust and sort the order of sub-images at different scales. The sub-image with maximal energy should be arranged in the first place in the same scale, the other sub-images should be arranged in descending order according to their energy parameter values, thus, the output sequence of sub-bands under two scales are formed.

$E_{k}=\frac{1}{m \times n} \sum_{i=1}^{m} \sum_{j=1}^{n}\left|w_{k}(i, j)\right|$

In formula (1), $m \times n$ is the size of sub-image, $w_{k}(i, j)$ is contourlet transform coefficient in the $k$ th sub-image.

(3) Coefficient matrix of each sub-image is quantified according to formula (2) to obtain a binary matrix. This process can be regarded as the enhancement of textural feature of image.

$I_{k}(i, j)=\left\{\begin{array}{cc}1 & \left|w_{k}(i, j)\right| \geq v_{k} \\ 0 & \text { else }\end{array}\right.$

Here, $v_{k}$ is the mean of energy of $k$ th sub-image. Then we up-sample all binary matrices to obtain the energy matrices with same size.

(4) We combine the values (0/1) into 12 bit binary numbers at the same location in energy matrices, which constitute a binary matrix $A$. Channel $0 \square 3$ are four 
sub-bands information resulting from 2-level contourlet transform, and channel $4 \square 11$ are eight sub-bands information resulting from 3-level contourlet transform.

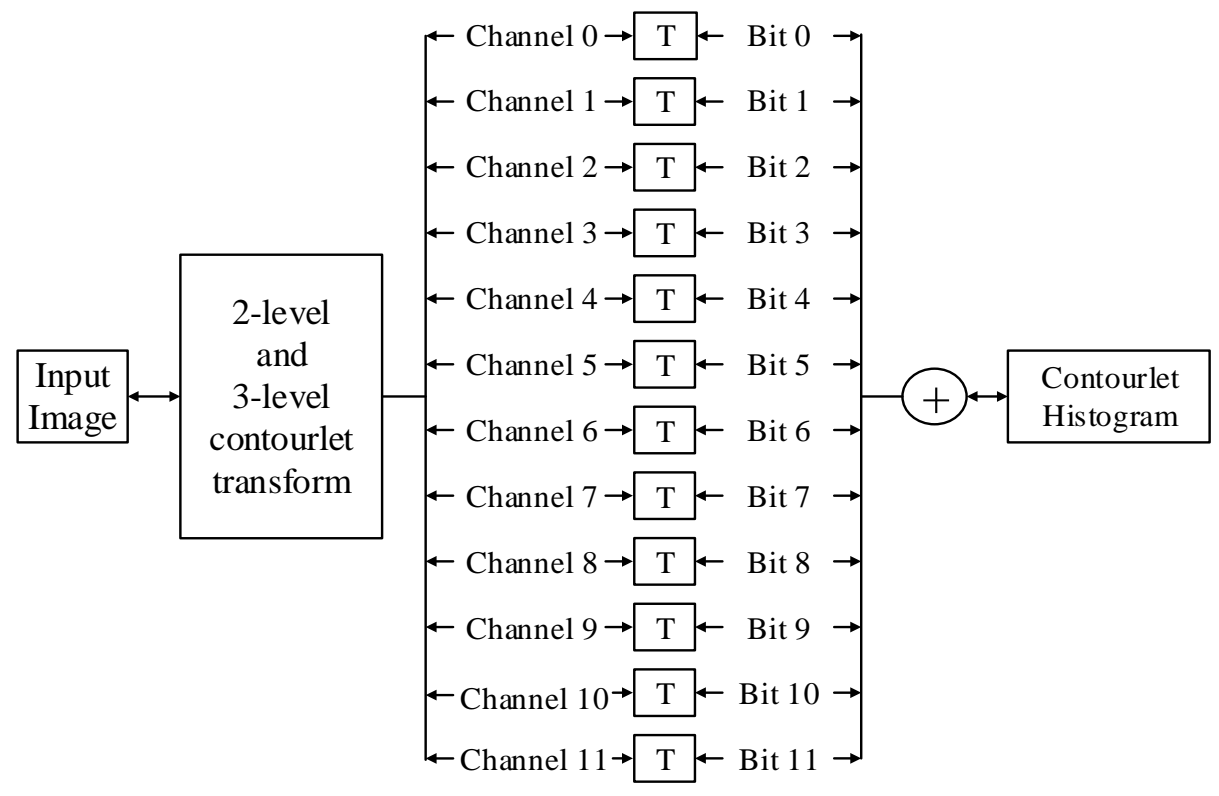

Figure 2. The Method of Constructing Contourlet Histograms

The method of constructing contourlet histogram is showed in Figure 2. Here, $T$ represents the binary quantification. We convert the dataset (that is the elements of matrix $A$ ) of 12 bit binary numbers to decimal numbers, and the range of these decimal numbers are $0 \square 4096$. The matrix of decimal numbers is denoted by $B$. We count the elements of matrix $B$ to obtain the contourlet histogram.

\section{Mean Shift for Contourlet Histogram}

Mean shift algorithm is a kind of non-parametric estimation algorithm based on density gradient. The algorithm makes the target converge to real position of target eventually by establishing target model, candidate target model, selecting an appropriate similarity function to measure the similarity between the two models, and calculating the maximum of the similarity to get mean shift vector of the target. Mean shift tracking algorithm is usually based on color probability distribution histogram of the target area, but this color feature are easily influenced by illumination change and the object that has similar color to the target. This paper uses contourlet histogram to extract textural feature of target image, and the pixel values of ordinary color histogram are replaced by the elements of matrix $B$ from section 2.2. Then we establish target model and candidate target model as the formula (3) and (4) respectively:

$$
\begin{aligned}
& \hat{q}_{u}=C \sum_{i=1}^{n} k\left(\left\|\frac{x_{0}-x_{i}}{h}\right\|^{2}\right) \delta\left[b\left(x_{i}\right)-u\right] \\
& \hat{p}_{u}=C_{h} \sum_{i=1}^{n} k\left(\left\|\frac{y-x_{i}}{h}\right\|^{2}\right) \delta\left[b\left(x_{i}\right)-u\right]
\end{aligned}
$$

In formula (3) and (4), $x_{0}$ is the element of center coordinate in matrix $B$; $x_{i}(i=1,2, \ldots, n)$ are other elements in matrix $\mathrm{B} ; k(g)$ is profile function of kernel function; $h$ is kernel bandwidth; $\delta(g)$ is the function of Kronecker delta to judge 
whether the element $x_{i}$ belongs to the $u$ th characteristic value; $C$ and $C_{h}$ are normalized constants, the calculation of them are as follows:

$$
\begin{aligned}
& C=\frac{1}{\sum_{i=1}^{n} k\left(\left\|\frac{x_{0}-x_{i}}{h}\right\|^{2}\right)} \\
& C_{h}=\frac{1}{\sum_{i=1}^{n} k\left(\left\|\frac{y-x_{i}}{h}\right\|^{2}\right)}
\end{aligned}
$$

Bhattacharyya coefficient is used to measure the similarity of target model and candidate target model as formula (7). We suppose that the center position of the target is $y_{0}$ in previous frame, we do a Taylor expansion for formula (7) at $\hat{p}_{u}\left(y_{0}\right)$, and calculate the maximum of $\hat{\rho}(y)$ through mean shift iteration. The corresponding mean shift vector is $M_{h, G}(y)$ by formula (8), the new position $y_{1}$ can be obtained by iteration, and $y_{1}$ is defined as formula (9).

$$
\begin{aligned}
& \hat{\rho}(y)=\sum_{u} \sqrt{\hat{p}_{u}(y) \hat{q}_{u}} \\
& M_{h, G}(y)=y_{1}-y_{0} \\
& y_{1}=\frac{\sum_{i=1}^{n} x_{i} w_{i} g\left(\left\|\frac{y_{0}-x_{i}}{h}\right\|^{2}\right)}{\sum_{i=1}^{n} w_{i} g\left(\left\|\frac{y_{0}-x_{i}}{h}\right\|^{2}\right)}
\end{aligned}
$$

Here,

$$
\begin{aligned}
& w_{i}=\sum_{i=1}^{n} \sqrt{\frac{\hat{q}_{u}}{\hat{p}_{u}\left(y_{0}\right)}} \delta\left[b\left(x_{i}\right)-u\right] \\
& g(x)=-k^{\prime}(x)
\end{aligned}
$$

\section{Variable Kernel Bandwidth Tracking Algorithm Based on Contourlet Histogram and Information Entropy}

A metric method was introduced into target tracking in literature [9], the method can measure the amount of information of multiscale images. In the literature, the size of kernel bandwidth was selected automatically according to the change of amount of information, and satisfying result was achieved. The method measured the magnitude of image information from the perspective of visual features of image, but it is complex to extract the visual features of image. While the features of images not only have visual features, but also have statistical features [15]. In this paper, from the perspective of statistical features of images, that is from the perspective of information theory, information entropy is used to measure the magnitude of image information, and according to the relationship between information and the size of target, a scale-update item is increased to the algorithm based on contourlet histogram with mean shift algorithm, finally, a variable kernel bandwidth tracking algorithm is designed based on contourlet histogram and information entropy. 


\subsection{Information Entropy of Image}

Information entropy of image is also called average amount of information, it only depends on the image itself. Any assumptions or prior knowledge is not required, preprocessing is also not needed, such as extracting feature points of the image.

Suppose that there is a $k$ level gray image $f(x, y)$, the probability of $i$ th $(i=1 \sim k)$ level gray is $p i$. Hence, the information entropy (the weighted average information) contained in this image is as follows:

$H=\sum_{i=0}^{k-1} p i \cdot \log \left(\frac{1}{p i}\right)=-\sum_{i=0}^{k-1} p i \cdot \log (p i)$

The meaning of information entropy is: the greater probability of image appears, the smaller information entropy it has, the less information content it transmits. Conversely, the less probability of image appears, the greater information entropy it has, the more information content it transmits.

As we all know, when we observe a picture in different distances, our feeling is different. For instance, the outline of the picture is only seen in far distance, then we can obtain much information form the image, and the average information is little, in other words, the information entropy is little; more details can be seen in near distance, then we can obtain less information, and the average information is much, the information entropy is much. Next, we research the relationship between image information entropy and the size of target.

\subsection{Relationship Between Image Information Entropy and the Size of Target}

When we research the relationship between information entropy and the size of target, an iterative search area in mean shift algorithm need to be defined. The area centers on the estimated value of target's location that is estimated by three points linear predictor (as showed in formula (13)), and the size of the area is $1+\alpha$ times larger than target template, so that the iterations in mean shift algorithm can be decreased, the influence of other objects on the target also can be eliminated.

$$
L(t)=\frac{1}{3}[4 L(t-1)+L(t-2)-2 L(t-3)]
$$

Here, the current frame is $t$ th frame, $L(t)$ is the position of target in $t$ th frame.

Literature [9] extended scale effect to its experiment, according to this basic idea, we select a toy that is as shown in Figure 3 to do a experiment, and the toy is regarded as a tracking target. In the experiment, we shoot a video with 50 frames from far to near, these area of video frame images are regarded as iterative search areas in mean shift algorithm, and the information entropy for each frame are calculated, then we manually set up a rectangular box for the target in each frame so that the target can be contained in the box, at the same time, the height of rectangle box should be measured. Figure 4(a) shows a graph that represents the changes of information entropy of the search area with the increase of the size of target, the horizontal axis represents the normalized height of rectangular box (i.e. the height of the object) in the current frame, and the vertical axis is the normalized value of information entropy. The figure shows that, when the size of target changes from small to large, the information entropy of search area changes from small to large. 

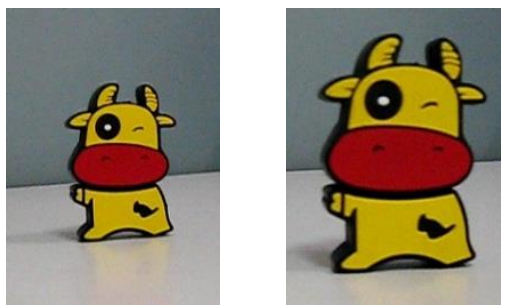

Figure 3. The Experiment Images of Toy from Far to Near

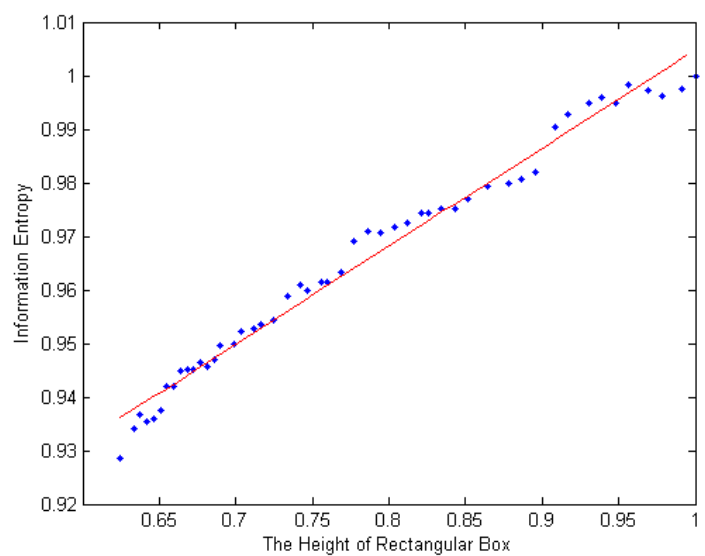

(a) The normalized curve graph about information entropy of search area with the increase of rectangular box's height

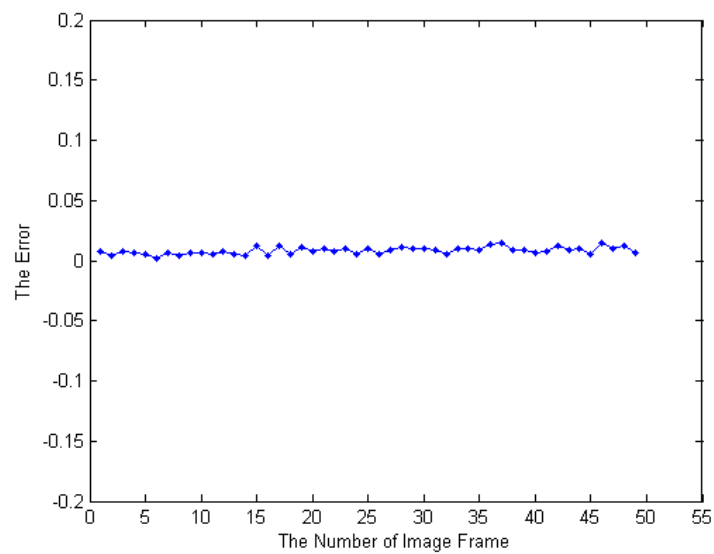

\section{Figure 4. The Experiment About Information Entropy and the Size of Target}

On the basis of the experiment, we calculate the proportion $S_{I}$ of information entropy of iterative search area of previous and current frame in mean shift algorithm respectively, and the proportion $S_{H}$ of the height of two rectangular boxes. Finally, the error that is represented as formula (14), the graph of error is showed as Figure 4(b).

$e=1+\lg \left(S_{I}\right)-S_{H}$

Figure 4(b) shows that the error is small, so kernel bandwidth can be updated according to the changes of information entropy. Hence, the relationship between information entropy of search area and the size of target is as follows:

$H \approx H_{0}\left(1+\lg \left(S_{I}\right)\right)$ 
Here, $H_{0}$ is the size of target in previous frame, $H$ is the size of target in current frame.

\subsection{Variable Kernel Bandwidth Tracking Algorithm}

Updating the size of tracking window plays a key role in the tracking algorithm. According to the relationship between information entropy and the size of target, a scale-update item is added to mean shift algorithm in this paper, and a new algorithm is designed that can change the size of tracking window with the changes of the size of target. The main idea of scale-update algorithm is: firstly, the estimated position of next frame is estimated by three points linear predictor, then we define an iterative search area whose size is $1+\alpha$ times larger than the size of target template and the center position of the area is the estimated position, so that the number of iteration of tracking algorithm can be decreased; secondly, we count information entropy $I_{k}$ and $I_{k+M}$ at interval of $M$ frames, and update the kernel bandwidth according to the proportion $S$ that represents the changes of the size of target, the representation of proportion $S$ is as formula (16).

$$
S=1+\lg \left(\frac{I_{k+M}}{I_{k}}\right)
$$

The algorithmic process of this paper is as follows:

(1) Initialize the number of frame $k=0$, define the proportion of changes of the size of target $S=0$, set the interval frame of kernel bandwidth updating $M$; set the size of the iterative search area is $1+\alpha$ times larger than the tracking window, the target's position $y_{0}$, calculate the contourlet histogram of target image and target model $q_{u}\left(y_{0}\right)$, and calculate entropy of the search area $I_{0}$;

(2) Fetch a video frame, $k=k+1$, estimate the position of moving target by three points linear predictor;

(3) If frame number $k$ is a multiple of $M$, calculate entropy $I_{i M}(i=1,2, \ldots)$ of the iterative search area in mean shift algorithm, then update the size of tracking window by formula (15); otherwise, go to step 4 directly;

(4) Obtain the candidate target area $A$ according to mean shift tracking algorithm, calculate the contourlet histogram about the candidate target area and its model $p_{u}(y)$, then calculate the position of candidate target $y_{1}$ by formula (9) and (10);

(5) Calculate the similarity between candidate target and target template, if $\rho\left(y_{1}\right)>\rho\left(y_{0}\right)$, then set $y_{1} \leftarrow\left(y_{1}+y_{0}\right) / 2$; If $\left\|y_{1}-y_{0}\right\| \leq \varepsilon$, stop calculating, set $y_{1}$ is the center of tracking window, the image in tracking window is chosen the target in current frame and set to target template, go to step 2; otherwise, set $y_{0} \leftarrow y_{1}$, go to step 4 .

\section{Experiment Results and Analysis}

To test the availability of the algorithm mentioned above, we use three algorithms to do tracking simulation experiment at Matlab 2014a, our test platform is a computer with Inter(R) Pentium(R) Dual CPU T3400 2.16GHz and its RAM is $2 \mathrm{G}$. The algorithms are the proposed algorithm, the tracking algorithm based on contourlet histogram (refers to CMS algorithm in next paper) in literature [6] and traditional mean shift algorithm based on color histogram (refers to TMS algorithm in next paper), the standard video sequences we choose have obvious size changes of target.

The testing sequences are from the dataset PETS2004 [16]. The resolution of original images is $384 \times 288$, the frame rate is $25 \mathrm{f} / \mathrm{s}$. We choose 640 frames video images 
(sequence 1), and the size of target is getting bigger in the sequence. In this experiment, the parameters that we select to update the tracking window are: $M=40, \alpha=0.2$. Figure 5(a), (b), (c) shows the tracking results of the proposed algorithm, CMS algorithm and TMS algorithm for augmented target respectively.

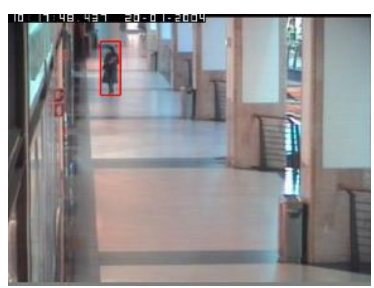

Frame 19

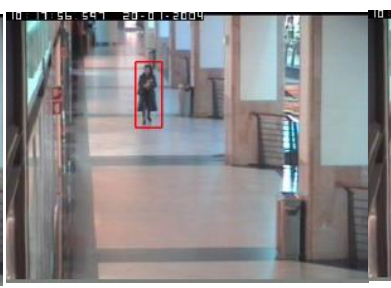

Frame 223

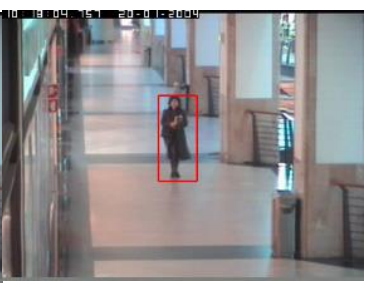

Frame 427

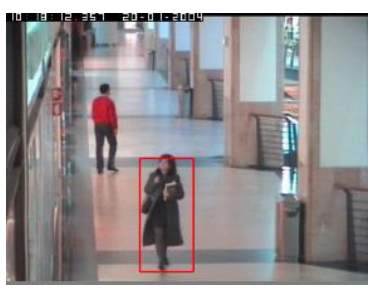

Frame 619

(a) The proposed algorithm

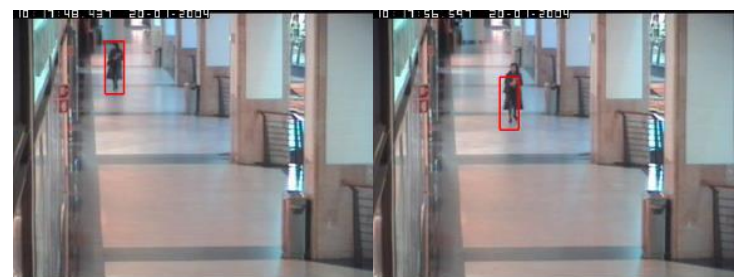

Frame 19
Frame 223

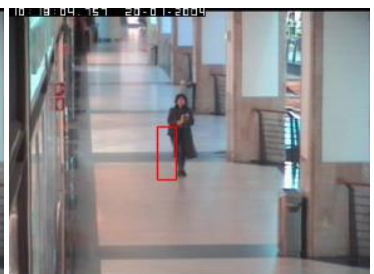

Frame 427

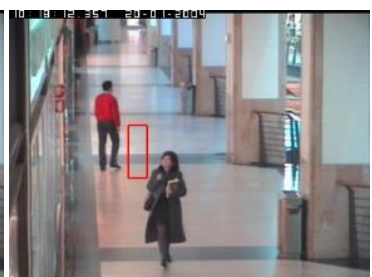

Frame 619

(b) CMS algorithm

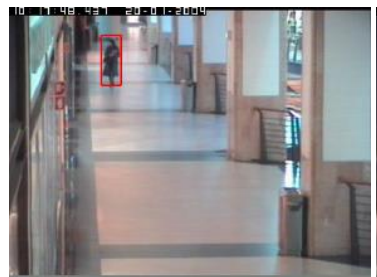

Frame 19

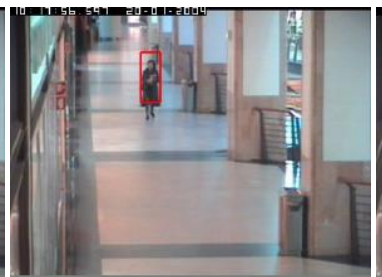

Frame 223

(c) TMS algorithm

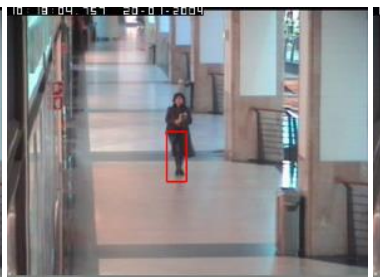

Frame 427

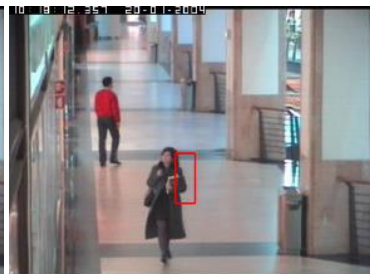

Frame 619

\section{Figure 5. The Results for Augmented Target}

We choose 400 frames video images (sequence 2), and the size of target is getting smaller in the sequence. In this experiment, the parameters that we select to update the tracking window are: $M=20, \alpha=0.2$. Figure 6(a), (b), (c) shows the tracking results of the proposed algorithm, CMS algorithm and TMS algorithm for decreasing target respectively.

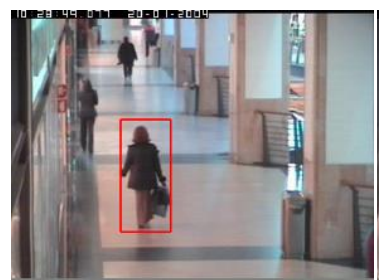

Frame 21

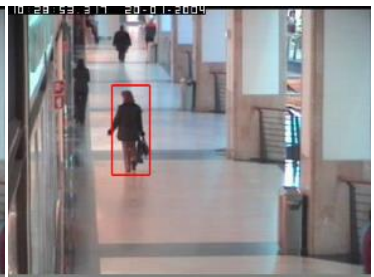

Frame 127

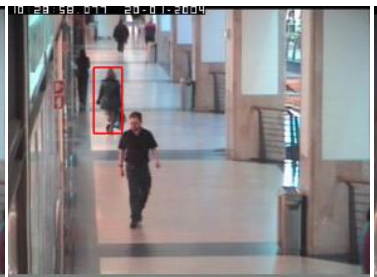

Frame 245

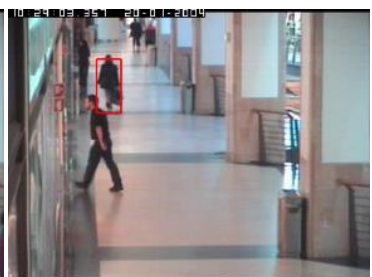

Frame 377

(a) The proposed algorithm 


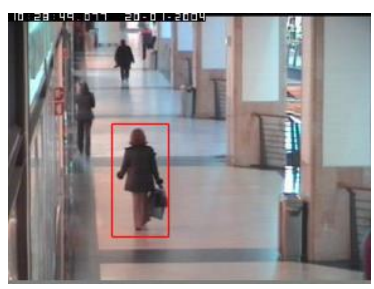

Frame 21

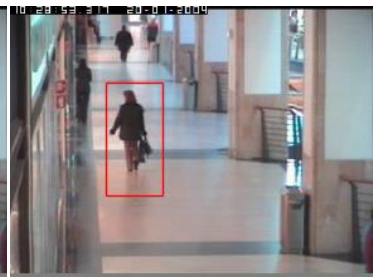

Frame 127

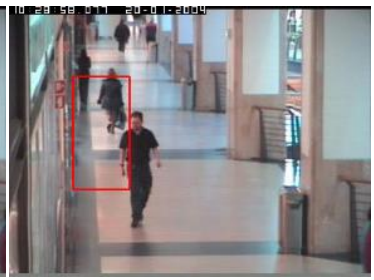

Frame 245

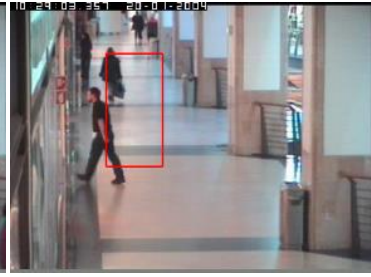

Frame 377

(b) CMS algorithm

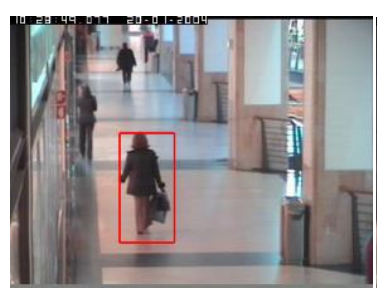

Frame 21

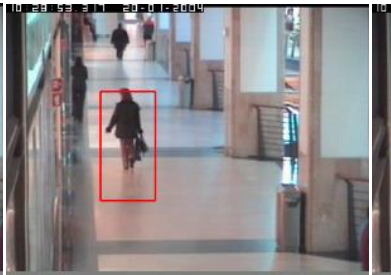

Frame 127

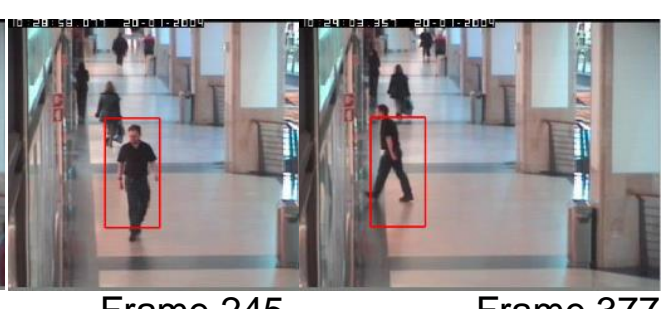

Frame 245

Frame 377

(c) TMS algorithm

Figure 6. The Results for Decreasing Target

In Figure 5 and Figure 6, the comparison results show that: when the change of the size of target is not obvious, the effect of tracking for three algorithms are nearly same. As the changes of the size of target don't considere in CMS algorithm and TMS algorithm, the tracking window are not updated by the two algorithms, the target templates happen to drift with the increasement of frame number, which lead to poor visual effect of tracking, even fail to track the target.

In Figure 6(b), (c), when the tracking window of video sequence 2 is large and there is other similar color non-object around the target, CMS algorithm has a better performance than TMS algorithm, because the CMS algorithm uses contourlet textural feature. Although the size of the target decreases in Figure 6(b) and its textural information also become weak, the target still has good outline information, the visual effect of tracking is not satisfying, the CMS algorithm does not fail to track the target. In Figure 6(c), the color of non-target object is similar to the color of target, the TMS algorithm fails to track the target. While the proposed algorithm extracts contourlet textural feature for target, and updates the kernel bandwidth in mean shift tracking algorithm to adapt the changes of target's size. When the target is interfered by other non-targets, the proposed algorithm still can realize robust tracking.

In order to verify tracking real-time of the proposed algorithm, we count average time-wasting per frame of the proposed algorithm, CMS algorithm and TMS algorithm, the real-time contrast results are showed in Table 1.

Table 1. Real-Time Comparison Between the Algorithms

\begin{tabular}{|c|c|c|}
\hline \multirow{2}{*}{ Items } & \multicolumn{2}{|c|}{ Time-wasting (frame/s) } \\
\cline { 2 - 3 } & $\begin{array}{c}\text { Target getting bigger } \\
\text { (video sequence 1) }\end{array}$ & $\begin{array}{c}\text { Target getting smaller } \\
\text { (video sequence 2) }\end{array}$ \\
\hline The proposed algorithm & 2.149 & 2.083 \\
\hline CMS algorithm & 1.921 & 1.757 \\
\hline TMS algorithm & 2.743 & 2.591 \\
\hline
\end{tabular}

From the Table 1, we can know that the tracking speed of TMS algorithm is most fast, because the algorithm only count the number of image pixels and its amount of 
calculation is few. The tracking speed of the proposed algorithm and CMS algorithm are slower than TMS algorithm's speed, because contourlet transform is needed to process by CMS algorithm and the proposed algorithm, then the contourlet coefficient is counted to obtain contourlet histogram. The position of target is estimated by the proposed algorithm, thus the iterations of mean shift algorithm are decreased. At the same time, we update the kernel bandwidth at interval $M$ frames, compared with other calculation, the time-wasting is very small. Hence, the speed of proposed algorithm is faster than the CMS algorithm.

In brief, although the tracking speed of traditional mean shift algorithm based on color histogram is fast, its tracking effect is not satisfying, when the algorithm is affected by illumination change and similar color objects, or the target has change of size in moving. The tracking speed of proposed algorithm is slower than TMS algorithm, but its kernel bandwidth can change with the changes of target's size, and it can resist the interference of other similar color non-objects.

\section{Conclusion}

In most tracking algorithms, color features which is often selected does not provide spatial information, and the target is often easily influenced by similar color non-object. In this paper, Contourlet histogram feature is chosen as textural model of the target to resist the interference, and mean shift tracking algorithm is chosen as tracking algorithm. While mean shift tracking algorithm with fixed kernel bandwidth fails to position the target accurately, when the size of target changes obviously. This paper introduces an item of updating the kernel bandwidth through studying and utilizing the relationship between information entropy and the size of target, and thereby a tracking algorithm with variable kernel bandwidth is put forward based on contourlet histogram and information entropy. Experimental results show that the proposed tracking algorithm can not only effectively overcome the interference of similar color non-target object in the scene, but also adapt to target's size change stably. Compared with the traditional mean shift tracking algorithm and the tracking algorithm based on contourlet histogram, the proposed algorithm has a better robustness. However, the algorithm cannot work well when the target is in a complex background, and we will make further effects to study the influence of background complexity on the relationship between information entropy and the size of target.

\section{Reference}

[1] F. Bousetouane, L. Dib and H. Snoussi, "Improved mean shift integrating texture and color features for robust real time object tracking", The Visual Computer, vol. 29, no. 3, (2013), pp. 155-170.

[2] A. Barley and C. Town, "Combinations of Feature Descriptors for Texture Image Classification", Journal of Data Analysis and Information Processing, vol. 2, no. 03, (2014), pp. 67-76.

[3] G. Wu, Z. M. Tang and J. Y. Yang, "Particle filter methods imported by typical texture 's characteristics in object tracking", Computer Engineering and Applications, vol. 47, no. 34, (2011), pp. 21-25.

[4] N. D. Minh and V. Martin, "Contourlets: A new directional multiresolution image representation". Conference Record of the Asilomar Conference on Signals, Systems and Computers, Pacific Grove, CA, USA, vol. 1, (2002) November 3-6, pp. 497-501.

[5] X. C. Tian, D. Yang and C. H. Du, "Image Retrieval Method Based on Color and Contourlet Histogram", Computer Engineering, vol. 36, no. 1, (2010), pp. 224-226.

[6] W. T. Guo and N. Cai, "Object tracking based on contourlet histogram", Laser \& infrared, vol. 42, no. 10, (2012), pp. 1177-1180.

[7] N. Cai, N. Zhu, W.T. Guo, BW-K. Ling, H. Wang and Q.Y. Dai, "Object tracking using mean shift for adaptive weighted-sum histograms", Circuits, Systems, and Signal Processing, vol. 33, no. 2, (2014), pp. 483-499.

[8] D. Comaniciu, V. Ramesh and P. Meer, "Kernel-based object tracking", Pattern Analysis and Machine Intelligence, IEEE Transactions on, vol. 25, no. 5, (2003), pp. 564-577.

[9] H. M. Qian, Y. Mao, J. Geng and Z. Wang, "Object tracking with self-updating tracking window", Intelligence and Security Informatics, Springer Berlin Heidelberg, (2007), pp. 82-93. 
[10] S. A. Mohammadi and M. R. Mahzoun, "A Novel Approach Coloured Object Tracker with Adaptive Model and Bandwidth using Mean Shift Algorithm", Signal \& Image Processing : An International Journal(SIPIJ), vol. 3, no. 3, (2012), pp. 1-15.

[11] Q. Lin, Y. X. Chen, S. T. Wang and Y. Z. Zhan, "MeanShift Tracking Algorithm with the Adaptive Bandwidth of the Target in Occlusions", Computer Science, vol. 37, no.8, (2010), pp. 273-275.

[12] F. Wang, G. L. Wei, B.Y. Wang and G. Y. Zou, "Mean-shift Tracking Algorithm with Adjustive Bandwidth Based on Partition", Opto-Electronic Engineering, vol. 41, no. 4, (2014), pp. 41-46.

[13] J. Wu, Z. Cui, P. Zhao and J. Chen, "Blocking Contourlet Transform: An Improvement of Contourlet Transformand Its Application to Image Retrieval", Journal of Computers, vol. 7, no. 9, (2012), pp. 2262-2268.

[14] Y. Choi, E. Sharifahmadian and S. Latifi, "Performance analysis of contourlet-based hyperspectral image fusion methods", International Journal on Information Theory, vol. 2, no. 1, (2013), pp. 1-14.

[15] Z. Y. Wang, Z. X. Cheng and S. J. Tang, "Information Meausures of scale-space based on visual characters", Journal of Image and Graphics, vol. 07, (2005), pp. 922-928.

[16] http://www-prima.inrialpes.fr/PETS04/caviar_data.html.

\section{Authors}
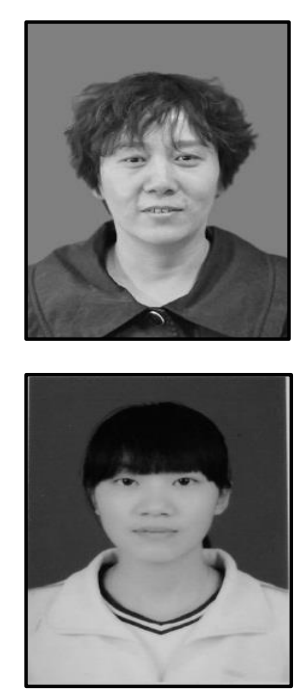

Rui Li works as a professor in School of Computer and Communication, LanZhou University of Technology. She received Master's Degree in computer application technology from Northwest Normal University in 2005.Research Interests: Intelligent Information Processing, Image Processing, Pattern Recognition and Digital Watermarking.

Wen Wang is pursuing Master's degree in Signal and Information Processing in LanZhou University of Technology. She received bachelor's degree from ChengDu University of Information Technology in 2012. Research Interests: Image Processing, Object tracking, Pattern Recognition. 